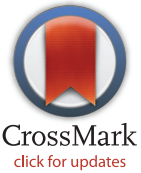

RESEARCH ARTICLE

\section{Slow Gait Speed and Rapid Renal Function Decline Are Risk Factors for Postoperative Delirium after Urological Surgery}

Tendo Sato ${ }^{1}$, Shingo Hatakeyama ${ }^{1}$ *, Teppei Okamoto ${ }^{1}$, Hayato Yamamoto', Shogo Hosogoe ${ }^{1}$, Yuki Tobisawa ${ }^{1}$, Tohru Yoneyama ${ }^{2}$, Eiji Hashiba ${ }^{3}$, Takahiro Yoneyama ${ }^{1}$, Yasuhiro Hashimoto ${ }^{2}$, Takuya Koie ${ }^{1}$, Kazuyoshi Hirota ${ }^{3}$, Chikara Ohyama ${ }^{1,2}$

1 Department of Urology, Hirosaki University Graduate School of Medicine, Hirosaki, Japan, 2 Department of Advanced Transplant and Regenerative Medicine, Hirosaki University Graduate School of Medicine, Hirosaki, Japan, 3 Department of Anesthesiology, Hirosaki University Graduate School of Medicine, Hirosaki, Japan

*shingoh@ @irosaki-u.c.jp

\section{Abstract \\ Objectives}

The aim of this study was to identify risk factors associated with postoperative delirium in patients undergoing urological surgery.

\section{Methods}

We prospectively evaluated pre- and postoperative risk factors for postoperative delirium in consecutive 215 patients who received urological surgery between August 2013 and November 2014. Preoperative factors included patient demographics, comorbidities, and frailty assessment. Frailty was measured by handgrip strength, fatigue scale of depression, fall risk assessment, and gait speed (the timed Get-up and Go test). Postoperative factors included types of anesthesia, surgical procedure, renal function and serum albumin decline, blood loss, surgery time, highest body temperature, and complications. Uni- and multivariate logistic regression analyses were performed to assess pre- and postoperative predictors for the development of postoperative delirium.

\section{Results}

Median age of this cohort was 67 years. Ten patients (4.7\%) experienced postoperative delirium. These patients were significantly older, had weak handgrip strength, a higher fall risk assessment score, slow gait speed, and greater renal function decline compared with patients without delirium. Multivariate analysis revealed slow gait speed (>13.0 s) and rapid renal function decline (>30\%) were independent risk factors for postoperative delirium.

\section{Conclusions}

Slow gait speed and rapid renal function decline after urological surgery are significant factors for postoperative delirium. These data will be helpful for perioperative patient management. This study was registered as a clinical trial: UMIN: R000018809. 


\section{Introduction}

Delirium after surgery is a big problem with consequences for patients and healthcare. Postoperative delirium incidences tend to increase in elderly patients[1] and varies from $3.1 \%$ to $54.9 \%$.[2-6] The older age is characteristic of aging of organs, increased occurrence of cognitive decline and functional impairment, and these are a large group of patients undergoing urological surgery. Incidence peaks of kidney, urinary bladder and prostate cancers were around 6080 years of age. Because the development of delirium in surgical patients has been associated with negative outcomes including a higher risk of postoperative mortality, $[2,7,8]$ geriatric assessment in older patients with genitourinary cancers, and management of postoperative delirium is an important issue. [9] However, only few studies included very few patients have evaluated postoperative delirium incidences after urological surgery. $[3,4,10]$ Postoperative delirium incidences are reported to be $8.8 \%$ in general urological surgery,[3] $29 \%$ in radical cystectomy,[4] and $21 \%$ in transurethral resection of prostate.[10] Identifying postoperative risk assessment is important in preventing delirium but remains challenging due to numerous causes. Several risk factors have been identified, including older age ( $\geq 65$ years), impaired outcome of cognitive and mental tests, poor nutritional status. However, the incidences of postoperative delirium in urological surgery vary widely because the etiology of delirium is multifactorial. In addition, it is challenging to apply all predictive factors into clinical practice without a well-trained geriatric care team with larger sample size. Therefore, an easy and simple method to evaluate frailty syndrome is required.

In 2004, the American Geriatric Society consensually defined "frailty" as "an excess vulnerability to stressors, with a reduced ability to maintain or regain homeostasis after a destabilizing event.[11] Physical parameters, such as gait speed, are an indicator of functional capacity and frailty syndrome. $[12,13]$ In recent years, there has been growing interest in measuring patients' gait speed at usual pace to screen for frailty.[14] This screening test is indeed quick, low-cost, and reproducible. Therefore, we hypothesized that physical frailty, including slow gait speed, may be associated with postoperative delirium in patients with urological surgery. The primary aim of the prospective study was to identify pre- and postoperative predictors for developing postoperative delirium after urological surgery. This study was registered as a clinical trial: UMIN: R000018809.

\section{Methods}

We prospectively evaluated frailty patients who admitted to our hospital for urological treatment between August 2013 and November 2014. In this period, 303 patients were admitted in our urological unit. Patients with non-surgical treatments $(n=82)$ were not included in this study. Of the remaining 221 patients, 2 patients did not undergo surgery due to comorbidities, and we excluded 4 patients due to insufficient data. Finally, we included a consecutive 215 patients (median age, 67 years) who received urological surgery. Delirium was diagnosed based on Diagnostic and Statistical Manual of Mental Disorders-V (DSM-V) criteria [15]. We screened five DSM-V criteria for delirium: A, There is a disturbance in attention and awareness (asking the same questions over and over and/or not be able to have a conversation). B, Delirium develops over a short period of time, typically hours to days. C, There is also another disturbance in cognition, such as in memory, orientation, language, and perception. $\mathrm{D}$, The disturbances in $(\mathrm{A})$ and $(\mathrm{C})$ are not better explained by another pre-existing, established, or evolving neurocognitive disorder (Essential to the diagnosis of delirium is that the patient can respond to "verbal stimulation"). E, There must also be evidence that the delirium is due to a direct physiological consequence of another medical condition, substance intoxication or withdrawal, or exposure to a toxin, or is due to multiple etiologies. If patients met any of these 
criteria, patients were diagnosed as a delirium. Well-experienced nurses assessed the DSM-V criteria once or twice a day during third postoperative day, and once a day afterwards. In addition, primary doctors investigated delirium at least once a day for all the length of hospital stay.

Inclusion criteria were major and minor surgeries for benign and malignant diseases. In major surgery, we included patients undergoing radical prostatectomy, radical or partial nephrectomy, radical cystectomy, laparoscopic adrenalectomy, renal transplantation, ureterocystoneostomy, retroperitoneal sarcoma resection, and repair of urethra-perineal fistula using a gracilis muscular flap after low anterior resection. Minor surgery included endoscopic transurethral resection of bladder tumor, transurethral cystolithotomy, and high orchiectomy. Exclusion criteria included admission for diseases with severe functional or cognitive impairment (who could not answer functional or cognitive questions), vision disorder or hearing loss, preexisting apparent dementia and cognitive loss, poor general health (ECOG performance status $>2$ ), medical conditions likely to result in death within a few months, or any other reasons because of which patients were unable to perform physical tests or answer the questionnaire on fatigue. In addition, obvious demented people were excluded at the outpatient clinic because these patients were not indicated for urological surgery. These criteria were assessed by well-experienced nurses and primary doctors.

\section{Ethics Statement}

This study was conducted in accordance with the ethical standards of the Declaration of Helsinki and approved by the ethical committee of Hirosaki University Graduate School of Medicine (authorization number: 2014-297). The participants in this study provide their written informed consent.

\section{Preoperative period}

Patients underwent a preoperative evaluation, which included patient characteristics and a frailty assessment, for 1 to 2 weeks before surgery. We assessed a frailty according to the modified Fried criteria,[16] which was focused on physical frailty and the questionnaire of fatigue. Physical frailty was measured by gait speed (the timed Get-up and Go test) and handgrip strength. The timed Get-up and Go test measures, in seconds, the time taken by an individual to stand up from a chair, walk a distance of $3 \mathrm{~m}$, turn, walk back to the chair, and sit down again. The presence of underlying depression and depressive symptoms were assessed using the fatigue scale of the Center for Epidemiologic Studies for Depression (CES-D). Furthermore, fall risk was investigated in this study. We used our original fall risk assessment scale,[17] which is a modified version of the Morse Fall Scale.[18] This fall risk assessment scale included age, past history of falls, vision or hearing disorder, functional disorder, activity, cognition, medication, and egestion. These assessments were performed at the first or second day of admission by several well-experienced nurses. We confirmed interrater reliability of the timed Get-up and Go test, handgrip strength and fall risk assessment.

Routine laboratory investigations were conducted including blood count, serum electrolyte and albumin levels, and liver and renal function tests. Renal function was evaluated using estimated glomerular filtration rate (eGFR), with a modified version of the abbreviated Modification of Diet in Renal Disease Study formula: eGFR mL/min $/ 1.73 \mathrm{~m}^{2}=194 \times \mathrm{sCr}^{-1.094} \times$ age $^{-0.287}(\times 0.739$, if female).[19] Nutritional status was evaluated by the Geriatric Nutritional Risk Index $\{\mathrm{GNRI}=[1.489+$ albumin $(\mathrm{g} / \mathrm{L})]+[41.7 \times$ body mass index/22] $\}$, developed as a tool for assessing nutritional risks, with the cut-off value of GNRI at $<92$.[20, 21]

The choices for anesthesia (general or regional) were decided by one or two senior supervisory doctors in our department, depending on the surgical risk, clinical condition, and 
comorbidities. The American Society of Anesthesiologists (ASA) classification [22] was used for assessing systemic comorbidity/performance, and it was obtained from the anesthesia chart.

\section{Anesthetic procedure and intraoperative period}

The anesthetic management and postoperative analgesia of patients was consistent and was not modified during this study. All major surgeries were conducted using general anesthesia, induced with remifentanil $(0.2-0.5 \mu \mathrm{g} / \mathrm{kg} / \mathrm{min})$, ketamine $(0.1-1.0 \mathrm{mg} / \mathrm{kg})$, and propofol (1-2 $\mathrm{mg} / \mathrm{kg})$. Tracheal intubation was facilitated with rocuronium bromide $(0.6 \mathrm{mg} / \mathrm{kg})$. Anesthesia was maintained with a continuous infusion of remifentanil $(0.2-0.5 \mu \mathrm{g} / \mathrm{kg} / \mathrm{min})$, ketamine $(0.5-1.0 \mathrm{mg} / \mathrm{kg} / \mathrm{h})$, and propofol $(4-6 \mathrm{mg} / \mathrm{kg} / \mathrm{h})$. Before the end of the surgery, intravenous morphine (5-10 mg) or fentanyl (2-4 $\mu \mathrm{g} / \mathrm{kg})$ was administered as boluses for an opioid rotation. Thereafter, intravenous patient-controlled analgesia using morphine (20-30 mg/day) or fentanyl (400-500 $\mu \mathrm{g} /$ day) with ketamine $(20 \mathrm{mg} /$ day) was followed to manage postoperative pain. General or regional anesthesia was also administered for minor surgeries. General anesthesia was similar to that used for open surgeries. Regional spinal anesthesia was induced by injecting 1.6-2.8 mL (body weight $\times 0.04 \mathrm{~mL}$ ) of hyperbaric bupivacaine $0.5 \%$ into the vertebral space. Moreover, intraoperative data were reviewed, including the type of surgery (major or minor), type of anesthesia (general or regional), and duration and blood loss of surgery.

\section{Postoperative period}

Postoperative data collection included the occurrence of delirium, complications, highest body temperature, renal function and serum albumin decline, and length of hospital stay. Postoperative renal function and serum albumin were evaluated 1 day after surgery. Complications were classified according to the Clavien-Dindo classification[23] and assessed in the postoperative period for up to 30 days.

\section{Statistical analysis}

Statistical analyses were conducted using GraphPad Prism version 5.03 (GraphPad software, Inc. La Jolla, CA, USA) and SPSS software package version 19.0 (SPSS, Chicago, IL, USA) with a $P$-value $<0.05$ (two-tailed) considered statistically significant. Quantitative variables were expressed as median, with quartiles 1 and $3(\mathrm{Q} 1$ and Q3). The between-group difference was statistically compared using the Student's $t$-test for normal distribution or the Mann-Whitney $U$ test for non-normal distribution. Categorical variables were reported as percentages (\%) and compared using Fisher's exact test. To determine predictive factors for postoperative delirium, patients were classified into two groups based on whether postoperative delirium occurred. Optimal cut-offs of age, Get-up and Go test, and fall risk assessment score were calculated by the formula $(1-\text { sensitivity })^{2}+(1-\text { specificity })^{2}$, with the help of receiver operator characteristics (ROC) curves.[24]

Here we separately analyzed pre- and postoperative variables. The preoperative risk factors for postoperative delirium were examined by uni- and multivariate logistic regression analyses including the following variables: age ( $>75$ years), sex (male), body mass index $\left(<20 \mathrm{~kg} / \mathrm{m}^{2}\right)$, medical history of type 2 diabetes, handgrip strength (male $<16 \mathrm{~kg}$, female $<18 \mathrm{~kg}$ ), Get-up and Go test ( $>13$ s), CES-D fatigue questionnaire (yes), fall risk assessment ( $>10$ points), nutritional status (GNRI < 92), and ASA status (score 3). For postoperative risk factors, highest body temperature recorded $\left(>38.0^{\circ} \mathrm{C}\right)$, postoperative complications (Clavien-Dindo $>1$ ), type of surgery (open), type of anesthesia (general), eGFR decline ( $>30 \%)$, serum albumin decline $(>30 \%)$, blood loss ( $>1000 \mathrm{~g})$, and duration of surgery $(>3 \mathrm{~h})$. Multivariate logistic 
regression was used to calculate odds ratios and $95 \%$ confidence intervals (CIs) after simultaneously controlling for potential confounders. Patients were categorized according to the number of independent predictors. The predictive accuracy of selected variables for postoperative delirium were evaluated by an area under the curve (AUC) derived from an ROC curve.

\section{Results}

\section{Characteristics of patients}

Of 215 surgical patients, 10 (4.7\%) developed postoperative delirium. Median age of the present study was 67 years old (interquartile range 63-75). According to types of surgical procedures, the occurrence of postoperative delirium was highest in nephrectomy and nephroureterectomy (30\%), followed by partial nephrectomy $8(10 \%)$, radical prostatectomy (10\%), radical cystectomy (10\%) and transurethral resection of bladder tumor (10\%). All episodes of delirium occurred within 3 days after surgery. Pre- and postoperative variables are listed in Table 1. Details of surgical procedures are shown in Table 2. Median eGFR change between preoperative and the day after surgery in radical prostatectomy, radical cystectomy, radical nephrectomy, partial nephrectomy, nephroureterectomy, other major surgeries (except for renal transplantation), and minor surgeries were $4.0 \%,-9.0 \%,-39 \%,-21 \%,-23 \%, 5 \%$, and $1 \%$, respectively. The minimal data set of the present study is available in S1 Dataset.

\section{Comparison of the patients}

Patients with delirium were significantly older $(P<0.001)$, had weaker handgrip strength $(P=0.002)$, slower gait speed in the timed Get-up and Go test $(P<0.001)$, and higher fall risk assessment $(P=0.002)$ than patients without delirium in preoperative factors. No patients had experienced previous postoperative delirium in this study. Except for a medical history of type 2 diabetes that showed marginal $(P=0.054)$ differences, there was no difference between patients with or without delirium in gender, body mass index, and nutritional status between the groups. In postoperative factors, no differences were observed between patients with or without delirium, except for rapid eGFR decline ( $P=0.002$; Fig $1 \mathrm{~A})$.

Optimal cut-off values of age, gait speed in the Get-up and Go test, and fall risk assessment scores were determined by ROC curves. Age greater than 75 years (AUC $=0.89, P<0.001$, $95 \%$ CI 0.82-0.95), gait speed slower than $13.0 \mathrm{~s}$ ( $\mathrm{AUC}=0.87, P<0.001,95 \% \mathrm{CI} 0.75-0.99$ ), and fall risk score higher than 10 points (AUC $=0.79, P=0.002,95 \% \mathrm{CI} 0.65-0.92$ ) were used for analysis in this study (Fig 1B).

\section{Uni- and multivariate logistic analysis}

Univariate logistic regression analysis showed older age ( $>75$ years), handgrip weakness, slower gait speed in the Get-up and Go test ( $>13.0$ s), CES-D fatigue status, higher score of fall risk assessment ( $>10$ points), and ASA score 3 were selected as preoperative significant factors, and rapid eGFR decline ( $>30 \%)$ was a postoperative risk factor for delirium. In multivariate analysis, slower gait speed in the Get-up and Go test $(P=0.008)$ and rapid eGFR decline $(P=0.003)$ were selected as an independent predictor for delirium (Table 3$)$. Age ( $>75$ years) showed marginal influences $(P=0.065)$ on delirium. Patients were categorized according to the number of independent predictors (scores $0-3$ ). The occurrence of postoperative delirium was 0 for $122(0 \%)$ patients with score 0 (= no risk factor), 1 for $68(1.4 \%)$ patients with score 1 (= 1 risk factor), 5 for 19 (26\%) patients with score 2 (2 risk factors), and 4 for 6 patients (67\%) with score 3 (3 risk factors) (Fig 1C) This risk classification indicated significant association with the occurrence of postoperative delirium $(P<0.0001)$. To evaluate the predictive accuracy 
Table 1. Patients' characteristics.

\begin{tabular}{|c|c|c|c|}
\hline Preoperative factors & No delirium & With delirium & $P$ value \\
\hline Number of patients, $n=$ & 205 & 10 & \\
\hline Age, years & $67(62,74)$ & $79(77,80)$ & $<0.001$ \\
\hline Gender (Male / Female), $\mathrm{n}=$ & $167 / 38$ & $8 / 2$ & 0.908 \\
\hline Body mass index, $\mathrm{kg} / \mathrm{m}^{2}$ & $22.9 \pm 2.7$ & $23.1 \pm 3.4$ & 0.665 \\
\hline Type 2 diabetes, $\mathrm{n}=$ & $17(8 \%)$ & $3(30 \%)$ & 0.054 \\
\hline Handgrip strength $(\mathrm{Kg})$ & $32(25,38)$ & $23(18,26)$ & 0.002 \\
\hline Get-up and Go test (seconds) & $9.0(7.5,11.0)$ & $15.8(13.6,17.3)$ & $<0.001$ \\
\hline CES-D Fatigue (Yes) & $18(9 \%)$ & $3(30 \%)$ & 0.062 \\
\hline Fall risk assessment score & $5.0(3.0,8.0)$ & $11.0(6.0,12.5)$ & 0.002 \\
\hline Nutritional status (GNRI) & $104(98,110)$ & $104(101,108)$ & 0.656 \\
\hline ASA score & $2(2,2)$ & $2(2,3)$ & 0.151 \\
\hline \multicolumn{4}{|l|}{ Types of disease, $n=$} \\
\hline Prostate Cancer & 86 & 1 & \\
\hline Bladder Cancer & 53 & 2 & \\
\hline Renal cell carcinoma & 29 & 4 & \\
\hline Adrenal tumor & 10 & 0 & \\
\hline Renal transplantation & 10 & 0 & \\
\hline Upper tract urothelial carcinoma & 7 & 3 & \\
\hline Others & 10 & 0 & \\
\hline Postoperative factors & No delirium & With delirium & $P$ value \\
\hline Type of anesthesia, $n=$ & & & 0.712 \\
\hline General & 176 & 9 & \\
\hline Regional (spinal or local) & 29 & 1 & \\
\hline Type of surgical procedure, $n=$ & & & 0.100 \\
\hline Open & 42 & 5 & \\
\hline Laparoscopic/Robotic & 122 & 4 & \\
\hline Minor & 41 & 1 & \\
\hline Renal function decline, $\%$ & $2(-8,16)$ & $33(28,39)$ & 0.002 \\
\hline Serum albumin decline, $\%$ & $21(15,25)$ & $24(19,26)$ & 0.164 \\
\hline Blood loss, $\mathrm{g}$ & $40(5-100)$ & $85(21,663)$ & 0.262 \\
\hline Surgery time, $\min$ & $159(112,188)$ & $145(131,180)$ & 0.655 \\
\hline Highest body temperature, ${ }^{\circ} \mathrm{C}$ & $37.5(37.2,37.8)$ & $38.0(37.8,38.0)$ & 0.191 \\
\hline Complications (Clavian-Dindo > 1) & $29(14 \%)$ & $1(10 \%)$ & 1.000 \\
\hline Hospital stay, days & $15.0(11.0,18.0)$ & $16.0(12.5,28.0)$ & 0.446 \\
\hline
\end{tabular}

Quantitative variables were expressed as median with quartile (Q1 and Q3).

doi:10.1371/journal.pone.0153961.t001

of the model, ROC curves were generated, and AUC was calculated. The AUC value in the risk classification was $0.95(P<0.001,95 \%$ CI, 0.90-1.00; Fig 1D).

\section{Discussion}

In this study, the occurrence of postoperative delirium was $4.7 \%$ (10/215 patients). Older age ( $>75$ years), slow gait speed ( $>13.0 \mathrm{~s}$ in Get-up and Go), and renal function decline $(>30 \%$ decline in eGFR the day after surgery) were identified as risk factors for postoperative delirium. Although many studies have described rates and risk factors for postoperative delirium in major surgeries, $[2,5]$ only few reports have investigated rates and risk factors for postoperative delirium in urological surgery. Postoperative delirium incidences are reported to be $29 \%$ in 
Table 2. Types of surgeries. We divided our urological surgery to two groups, major and minor. Surgeries under general anesthesia and local or spinal anesthesia were regarded as major and minor, respectively.

\begin{tabular}{lcc}
\hline & No delirium & With delirium \\
\hline 1. Major surgeries & & $1(20 \%)$ \\
Radical prostatectomy & $86(42 \%)$ & 0 \\
\hline Open & 7 & 1 \\
\hline Robotic & 79 & $1(10 \%)$ \\
Radical cystectomy & $14(7 \%)$ & 1 \\
\hline Open & 12 & 0 \\
\hline Robotic & 2 & $3(30 \%)$ \\
\hline Radical nephrectomy & $28(14 \%)$ & 2 \\
\hline Open & 8 & 1 \\
\hline Laparoscopic & 20 & $1(10 \%)$ \\
\hline Partial nephrectomy & $11(5.4 \%)$ & 0 \\
\hline Open & 7 & 1 \\
\hline Robotic & 4 & $3(30 \%)$ \\
Nephroureterectomy & $7(3.4 \%)$ & 2 \\
\hline Open & 1 & 1 \\
\hline Laparoscopic & 6 & $0(0 \%)$ \\
Others & $18(9 \%)$ & 0 \\
\hline Open procedures & 3 & 0 \\
\hline Laparoscopic Adrenalectomy & 10 & 0 \\
\hline Renal transplantation & 4 & 0 \\
\hline Laparoscopic lymph node biopsy & 1 & 0 \\
\hline 2. Minor surgeries & $10 \%)$ \\
\hline Transurethral resection of bladder tumor & $(20 \%)$ & 1 \\
\hline Orchiectomy & 39 & 0 \\
\hline Transurethral lithotripsy of bladder & 1 & 0 \\
\hline
\end{tabular}

doi:10.1371/journal.pone.0153961.t002

radical cystectomy,[4] 21\% in transurethral resection of prostate,[10] and $8.8 \%$ general urological surgery,[3] which were relatively higher than the present study. In those studies, delirium was screened and diagnosed using the Confusion Assessment Method, which may explain the higher incidence of delirium in comparison to the present study. Huge methodological differences among studies are important drawbacks to diagnose delirium. Therefore, the lack of acceptable explanation for the differences among various studies is an important limitation. Risk factors identified in those studies were older age ( $\geq 65$ years), impairment in the instrumental activities of daily living, poor clock drawing test, geriatric depression scale, a previous history of delirium, and mental status examinations. However, all predictive factors cannot be applied into clinical practice without a well-trained geriatric care team, and therefore, an easy and simple method to evaluate frailty syndrome is required.

Recent systematic review demonstrated the predictive values of a slow gait speed in early death, disability, falls and hospitalization/institutionalization in the elderly (65 and older).[25] They recommended gait speed test for screening of sarcopenia in the elderly, which is a central element in the pathophysiology of frailty. The International Society of Geriatric Oncology (SIOG) also recommends the gait speed alone or included in composite tests, such as the timed Get-up and Go test and the Short Physical Performance Battery (SPPB) test, to assess functional status and mobility in a comprehensive geriatric assessment. [25] In addition, previous study suggested slow gait speed (the timed Get-up and Go test, $>20 \mathrm{~s}$ ) was significantly associated with the occurrence of early death within 6 months of first-line chemotherapy in 
A
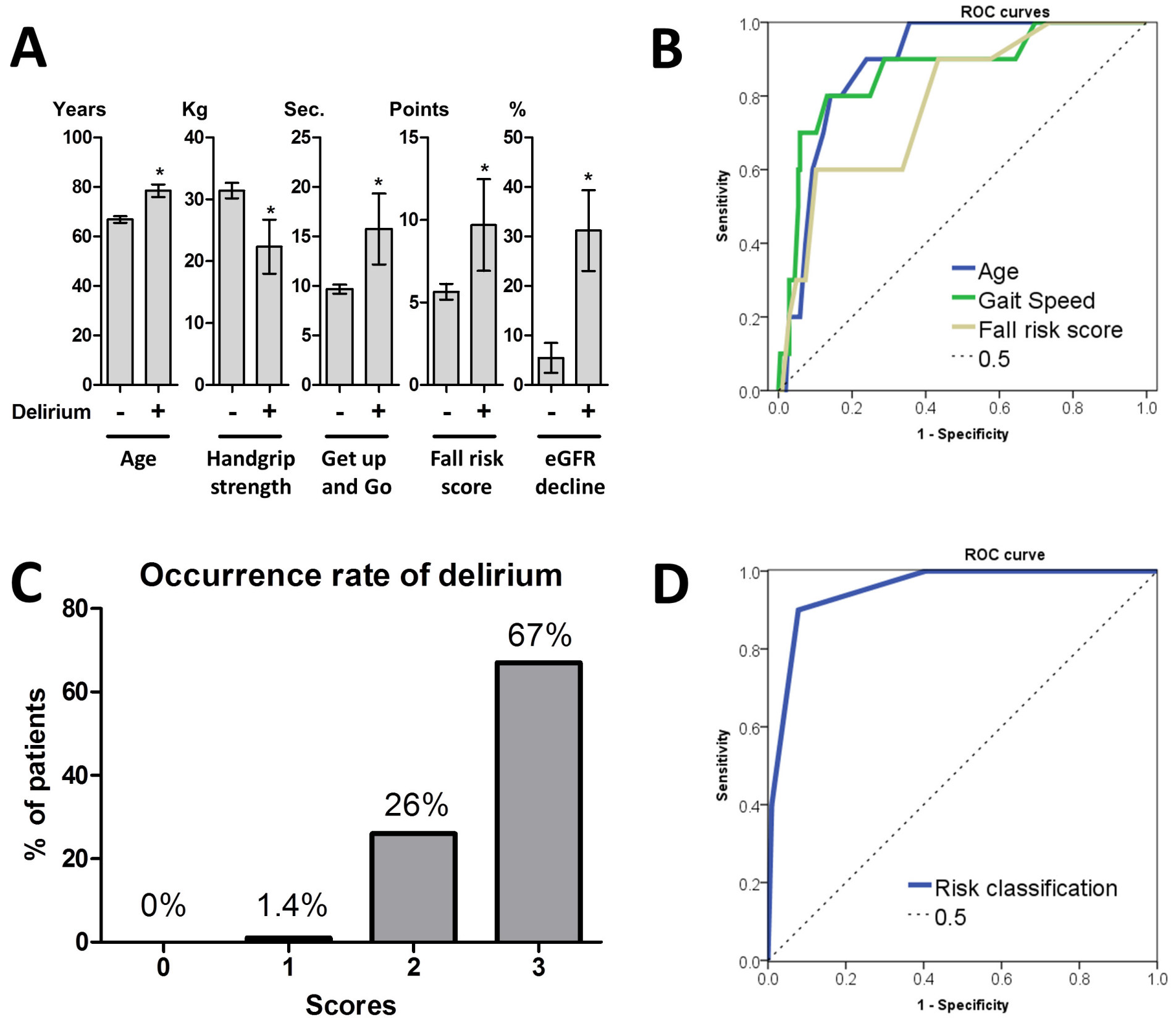

Fig 1. Predictive value of the risk stratification. A: Patients characteristics that were significantly different between the groups $(*, P<0.01)$. Error bar demonstrated $95 \% \mathrm{CI}$. B: ROC curve analysis to determine optimal cut-off values of age, gait speed in Get-up and Go test, and fall risk assessment scores. The AUC values were 0.89 in age, 0.87 in Get-up and Go test, and 0.79 in fall risk assessment score. The optimal cut-off values were age older than 75 years, slower than $13.0 \mathrm{~s}$ in the Get-up and Go test, and higher than 10 points in fall risk assessment score. C: Patients were categorized according to the number of independent predictors (>75 years old, Get-up and Go $>13.0$ s, eGFR decline $>30 \%$ ) for postoperative delirium (scores $0-3$ ). The occurrence of postoperative delirium was $0 \%$ in score $0,1.4 \%$ in score $1,26 \%$ in score 2 , and $67 \%$ in score $3(P<0.001)$. D: Predictive accuracy of selected three factors by the ROC curve showed that the AUC value was $0.952(P<0.001,95 \% \mathrm{Cl} 0.902-1.00)$.

doi:10.1371/journal.pone.0153961.g001

heterogeneous elderly cancer population,[26] and postoperative delirium in major abdominal surgeries.[2] Therefore, gait speed is an important indicator of functional capacity and general frailty,[13] and it is positively correlated with cognitive frailty in elderly patients.[2, 27, 28] Based on these findings, we employed the timed Get-up and Go test as a predictor for frailty syndrome that could evaluate both gait speed and complex maneuvers. As expected, the results 
Table 3. Uni- and multivariate logistic regression analysis of independent risk factors for incident of postoperative delirium.

\begin{tabular}{|c|c|c|c|c|c|c|c|}
\hline & \multirow[b]{2}{*}{ Preoperative risk factors } & \multicolumn{3}{|c|}{ Univariate analysis } & \multicolumn{3}{|c|}{ Multivariate analysis } \\
\hline & & $P$ value & OR & $95 \% \mathrm{Cl}$ & $P$ value & OR & $95 \% \mathrm{Cl}$ \\
\hline Age & $>75$ yrs old & 0.002 & 28.65 & $3.54-231$ & 0.065 & 10.14 & $0.86-119$ \\
\hline Gender & Male & 0.908 & 0.91 & $0.19-4.46$ & 0.398 & 2.60 & $0.28-24.0$ \\
\hline Body mass index & Less than $20 \mathrm{~kg} / \mathrm{m}^{2}$ & 0.745 & 1.30 & $0.26-6.41$ & 0.758 & 1.51 & $0.11-21.2$ \\
\hline Type 2 diabetes & Positive & 0.034 & 4.74 & $1.12-20.0$ & 0.328 & 2.84 & $0.35-22.9$ \\
\hline Handgrip strength & Male $<16 \mathrm{~kg}$, Female $<18 \mathrm{~kg}$ & 0.004 & 6.81 & $1.83-25.4$ & 0.125 & 4.10 & $0.67-25.0$ \\
\hline Get-up and go & $>13 \mathrm{sec}$ & 0.000 & 35.05 & $6.98-176$ & 0.008 & 12.49 & $1.95-80.2$ \\
\hline CES-D fatigue & Positive & 0.042 & 4.45 & $1.06-18.72$ & 0.118 & 4.80 & $0.67-34.3$ \\
\hline Fall risk assessment & $>10$ & 0.005 & 6.59 & $1.77-24.5$ & 0.916 & 1.10 & $0.18-6.59$ \\
\hline Nutritional status & GNRI $<92$ & 0.802 & 0.156 & $0.156-11.0$ & 0.600 & 2.81 & $0.06-133$ \\
\hline \multirow[t]{3}{*}{ ASA score } & Score 3 & 0.044 & 3.89 & $1.04-14.6$ & 0.939 & 1.07 & $0.18-6.25$ \\
\hline & & \multicolumn{3}{|c|}{ Univariate analysis } & \multicolumn{3}{|c|}{ Multivariate analysis } \\
\hline & Postoperative risk factors & $P$ value & OR & $95 \% \mathrm{Cl}$ & $P$ value & OR & $95 \% \mathrm{Cl}$ \\
\hline Highest body temperature & $>38.0^{\circ} \mathrm{C}$ & 0.609 & 1.52 & $0.31-7.50$ & 0.660 & 0.65 & $0.09-4.52$ \\
\hline Clavien-Dindo grade & $>1$ & 0.713 & 0.67 & $0.08-5.52$ & 0.581 & 0.53 & $0.06-5.00$ \\
\hline Types of surgery & Open & 0.053 & 3.56 & $0.99-12.8$ & 0.215 & 2.86 & $0.54-15.1$ \\
\hline Anesthesia & General & 0.713 & 1.48 & $0.18-12.2$ & 0.712 & 0.64 & $0.06-6.75$ \\
\hline Renal function decline & $>30 \%$ in eGFR & 0.002 & 8.42 & $2.25-31.6$ & 0.003 & 9.14 & $2.15-38.8$ \\
\hline Serum albumin decline & $>30 \%$ & 0.167 & 3.17 & $0.62-16.3$ & 0.602 & 1.75 & $0.21-14.3$ \\
\hline Blood loss & $>1000 \mathrm{~g}$ & 0.081 & 4.41 & $0.83-23.3$ & 0.847 & 1.29 & $0.10-17.5$ \\
\hline Operation time & $>3$ hours & 0.910 & 0.92 & $0.23-3.68$ & 0.427 & 0.50 & $0.09-2.76$ \\
\hline
\end{tabular}

doi:10.1371/journal.pone.0153961.t003

showed that slow gait speed $(>13.0 \mathrm{~s}$ ) was a strong predictor for postoperative delirium. This is the first report that identifies the cutoff value at $13 \mathrm{~s}$ in urological surgery. This may be caused by, at least in part, a study population difference between Western countries and Japan.

Because definitive evidence regarding gait speed for delirium is yet to be established, a further larger scale study is required to address the issue of optimal cut-off values.

We first found that postoperative delirium is significantly frequent in nephrectomy or nephroureterectomy patients (in total, $60 \%, P=0.003$, Fisher's exact test). Therefore, we hypothesized that the loss of rapid renal function may play an important role in the occurrence of postoperative delirium. We then investigated eGFR change between before and 1 day after surgery. The results indicated that rapid eGFR decline was significantly greater in the delirium group than that in the no-delirium group. In logistic regression analysis, eGFR decline $>30 \%$ was selected as a predictor for postoperative delirium. To our knowledge, this is the first report to identify the relationship between rapid renal function decline and postoperative delirium in urological surgery. Although our study could not address the background it can be hypothesized that the acute decline of renal function may induce an accumulation of anesthetizing agents, opioids, and/or metabolic substances after unilateral nephrectomy. Measuring the concentration of anesthetizing agent may help stratifying the risk for postoperative delirium. Further study is required to address these issues.

Next, we constructed a predictive model of postoperative delirium using independently significant factors. Because older age was reported as a strong predictor for postoperative delirium, we included older age ( $>75$ years) for the predictive model of postoperative delirium. Based on these selected three factors, we stratified patients into four categories according to the number of independent predictors (scores $0-3$ ). The occurrence of postoperative delirium was significantly higher in high score patients than that in lower score patients $(P<0.001)$. The AUC value of 0.95 indicated the high predictive accuracy of the risk stratification. 
There are several limitations which should be reported. First, there is no data for cognitive measurement, including the Mini-mental Status Examination and lack of dementia diagnosis due to the lack of a well-trained geriatric care team at our hospital. We only used five delirium criteria to diagnose delirium. Based on five checklists, we were able to evaluate whether delirium was positive or negative, although not all nurses and doctors were well trained. However, because the investigators who diagnose delirium were not specialist for geriatric screening, it is difficult to ensure the quality of each assessment. Therefore, we could not exclude under detection of postoperative delirium, and systematically measure all types of delirium and severity.

Second, obvious demented people were excluded at the out-patient clinic because these patients were not indicated for urological surgery. The reason for the low delirium incidence in the present study might be the exclusion of demented people from surgery at out-patient clinic. The differences of indication of surgery among studies might be important drawbacks in incidence rates of delirium.

Third, the findings are not directly generalizable to other settings because of a single center study. The statistical power was inadequate for addressing the independent risk factors in this study due to the low occurrence of postoperative delirium. In addition, decision to set the cut-off value for the timed Get-up and Go test at $13.0 \mathrm{sec}$ are not directly generalizable to other settings. Because no study assessed the implication of the timed Get-up and Go test in urological surgery and Japanese population, we statistically established our optimal cut-off value. Fourth, although we guaranteed that both the anesthetic management and the postoperative analgesia of patients were consistent, and it was not modified during this study, we could not address the unmeasurable confounders including anesthetic procedures, dosages of anesthetic agents, opioids, and dosages. Fifth, we did not assess the long-term complications of delirium. Because delirium is associated with medium and long-term poor outcomes, further investigations are necessary to assess the accuracy of delirium diagnosis and patients outcomes.

Despite these limitations, to our knowledge, this is the first report to investigate the relationship among gait speed, renal function decline, and occurrence of postoperative delirium. Our finding demonstrated that clinicians should be take special care in elderly patients with slow gait speed and uninephrectomy to address postoperative delirium. In addition, our data suggested that rapid renal function decline may have negative impact on the occurrence of postoperative delirium in other surgeries. Further validation studies that include cognitive measurement tools and other types of surgeries are warranted.

In conclusion, slow gait speed and rapid renal function decline after urological surgery independently associated with postoperative delirium. Although these factors must be validated in a larger cohort, our results will be helpful for perioperative patient management.

The minimal data set of the present study is available in S1 Dataset (Excel file).

\section{Supporting Information}

S1 Dataset. The minimal data set (Microsoft Excel file) of the present study. (XLSX)

\section{Author Contributions}

Conceived and designed the experiments: CO. Performed the experiments: TS TO HY S. Hosogoe YH Takahiro Yoneyama. Analyzed the data: S. Hatakeyama. Contributed reagents/materials/analysis tools: Thoru Yoneyama YH TK YT. Wrote the paper: S. Hatakeyama. Supervision: TK EH KH. 


\section{References}

1. O'Regan NA, Fitzgerald J, Timmons S, O'Connell H, Meagher D. Delirium: a key challenge for perioperative care. International journal of surgery (London, England). 2013; 11(2):136-44. Epub 2013/01/02. doi: 10.1016/j.ijsu.2012.12.015 PMID: 23277227.

2. Brouquet A, Cudennec T, Benoist S, Moulias S, Beauchet A, Penna C, et al. Impaired mobility, ASA status and administration of tramadol are risk factors for postoperative delirium in patients aged 75 years or more after major abdominal surgery. Annals of surgery. 2010; 251(4):759-65. Epub 2010/03/ 13. doi: 10.1097/SLA.0b013e3181c1cfc9 PMID: 20224380.

3. Tognoni P, Simonato A, Robutti N, Pisani M, Cataldi A, Monacelli F, et al. Preoperative risk factors for postoperative delirium (POD) after urological surgery in the elderly. Archives of gerontology and geriatrics. 2011; 52(3):e166-9. Epub 2010/11/19. doi: 10.1016/j.archger.2010.10.021 PMID: 21084123.

4. Large MC, Reichard C, Williams JT, Chang C, Prasad S, Leung Y, et al. Incidence, risk factors, and complications of postoperative delirium in elderly patients undergoing radical cystectomy. Urology. 2013; 81(1):123-8. Epub 2012/11/17. doi: 10.1016/j.urology.2012.07.086 PMID: 23153950.

5. Smulter N, Lingehall HC, Gustafson $Y$, Olofsson B, Engstrom KG. Delirium after cardiac surgery: incidence and risk factors. Interactive cardiovascular and thoracic surgery. 2013; 17(5):790-6. Epub 2013/ 07/28. doi: 10.1093/icvts/ivt323 PMID: 23887126; PubMed Central PMCID: PMCPMC3805209.

6. Chung KS, Lee JK, Park JS, Choi CH. Risk factors of delirium in patients undergoing total knee arthroplasty. Archives of gerontology and geriatrics. 2015; 60(3):443-7. Epub 2015/02/24. doi: 10.1016/j. archger.2015.01.021 PMID: 25704295.

7. Maniar HS, Lindman BR, Escallier K, Avidan M, Novak E, Melby SJ, et al. Delirium after surgical and transcatheter aortic valve replacement is associated with increased mortality. The Journal of thoracic and cardiovascular surgery. 2015. Epub 2016/01/18. doi: 10.1016/j.jtcvs.2015.10.114 PMID: 26774165.

8. Postoperative delirium in older adults: best practice statement from the American Geriatrics Society. Journal of the American College of Surgeons. 2015; 220(2):136-48 e1. Epub 2014/12/24. doi: 10.1016/ j.jamcollsurg.2014.10.019 PMID: 25535170.

9. Droz J- P, Audisio RA, International Society of Geriatric Oncology. Management of urological cancers in older people. London: Springer; 2013. Available: http://BN7ZQ5YK2C.search.serialssolutions.com/ $? \mathrm{~V}=1.0 \& \mathrm{~L}=\mathrm{BN7ZQ5YK2C} \& \mathrm{~S}=\mathrm{JC} \& \& \mathrm{C}=\mathrm{TC} 0000767082 \& \mathrm{~T}=$ marc $\& \mathrm{tab}=\mathrm{BOOKS}$.

10. Tai S, Xu L, Zhang L, Fan S, Liang C. Preoperative risk factors of postoperative delirium after transurethral prostatectomy for benign prostatic hyperplasia. International journal of clinical and experimental medicine. 2015; 8(3):4569-74. Epub 2015/06/13. PMID: 26064386; PubMed Central PMCID: PMCPMC4443220.

11. Walston J, Hadley EC, Ferrucci L, Guralnik JM, Newman AB, Studenski SA, et al. Research agenda for frailty in older adults: toward a better understanding of physiology and etiology: summary from the American Geriatrics Society/National Institute on Aging Research Conference on Frailty in Older Adults. Journal of the American Geriatrics Society. 2006; 54(6):991-1001. Epub 2006/06/17. doi: 10. 1111/j.1532-5415.2006.00745.x PMID: 16776798.

12. Byard RW. Frailty syndrome-Medicolegal considerations. Journal of forensic and legal medicine. 2015; 30:34-8. Epub 2015/01/28. doi: 10.1016/j.jflm.2014.12.016 PMID: 25623193.

13. Verghese J, Holtzer R, Lipton RB, Wang C. Quantitative gait markers and incident fall risk in older adults. The journals of gerontology Series A, Biological sciences and medical sciences. 2009; 64 (8):896-901. Epub 2009/04/08. doi: 10.1093/gerona/glp033 PMID: 19349593; PubMed Central PMCID: PMCPMC2709543.

14. Abellan van Kan G, Rolland $Y$, Houles M, Gillette-Guyonnet S, Soto M, Vellas B. The assessment of frailty in older adults. Clinics in geriatric medicine. 2010; 26(2):275-86. Epub 2010/05/26. doi: 10.1016/ j.cger.2010.02.002 PMID: 20497846.

15. American Psychiatric Association., American Psychiatric Association. DSM-5 Task Force. Diagnostic and statistical manual of mental disorders: DSM-5. 5th ed. Arlington VA: American Psychiatric Association; 2013. xliv, 947 p. p.

16. Saum KU, Muller H, Stegmaier C, Hauer K, Raum E, Brenner H. Development and evaluation of a modification of the Fried frailty criteria using population-independent cutpoints. Journal of the American Geriatrics Society. 2012; 60(11):2110-5. Epub 2012/10/10. doi: 10.1111/j.1532-5415.2012.04192.x PMID: 23043490.

17. Sugiyama R. Evidence based fall prevention; risk factors and assessment tools. Nursing. $2005 ; 25$ (14):32-9.

18. Morse JM, Morse RM, Tylko SJ. Development of a Scale to Identify the Fall-Prone Patient. Canadian Journal on Aging/La Revue canadienne du vieillissement. 1989; 8(04):366-77. doi: 10.1017/ S0714980800008576 
19. Matsuo S, Imai E, Horio M, Yasuda Y, Tomita K, Nitta K, et al. Revised equations for estimated GFR from serum creatinine in Japan. American journal of kidney diseases: the official journal of the National Kidney Foundation. 2009; 53(6):982-92. Epub 2009/04/03. doi: 10.1053/j.ajkd.2008.12.034 PMID: 19339088.

20. Bouillanne O, Morineau G, Dupont C, Coulombel I, Vincent JP, Nicolis I, et al. Geriatric Nutritional Risk Index: a new index for evaluating at-risk elderly medical patients. The American journal of clinical nutrition. 2005; 82(4):777-83. Epub 2005/10/08. PMID: 16210706.

21. Yamada K, Furuya R, Takita T, Maruyama Y, Yamaguchi Y, Ohkawa S, et al. Simplified nutritional screening tools for patients on maintenance hemodialysis. The American journal of clinical nutrition. 2008; 87(1):106-13. Epub 2008/01/08. PMID: 18175743.

22. Cullen DJ, Apolone G, Greenfield S, Guadagnoli E, Cleary P. ASA Physical Status and age predict morbidity after three surgical procedures. Annals of surgery. 1994; 220(1):3-9. Epub 1994/07/01. PMID: 8024356; PubMed Central PMCID: PMCPMC1234280.

23. Dindo D, Demartines N, Clavien PA. Classification of surgical complications: a new proposal with evaluation in a cohort of 6336 patients and results of a survey. Annals of surgery. 2004; 240(2):205-13. Epub 2004/07/27. PMID: 15273542; PubMed Central PMCID: PMCPMC1360123.

24. Akobeng AK. Understanding diagnostic tests 3 : Receiver operating characteristic curves. Acta paediatrica (Oslo, Norway: 1992). 2007; 96(5):644-7. Epub 2007/03/23. doi: 10.1111/j.1651-2227.2006. 00178.x PMID: 17376185.

25. Pamoukdjian F, Paillaud E, Zelek L, Laurent M, Levy V, Landre T, et al. Measurement of gait speed in older adults to identify complications associated with frailty: A systematic review. Journal of geriatric oncology. 2015; 6(6):484-96. Epub 2015/09/13. doi: 10.1016/j.jgo.2015.08.006 PMID: 26362356.

26. Soubeyran P, Fonck M, Blanc-Bisson C, Blanc JF, Ceccaldi J, Mertens C, et al. Predictors of early death risk in older patients treated with first-line chemotherapy for cancer. Journal of clinical oncology: official journal of the American Society of Clinical Oncology. 2012; 30(15):1829-34. Epub 2012/04/18. doi: 10.1200/jco.2011.35.7442 PMID: 22508806.

27. Gillain S, Warzee E, Lekeu F, Wojtasik V, Maquet D, Croisier JL, et al. The value of instrumental gait analysis in elderly healthy, $\mathrm{MCl}$ or Alzheimer's disease subjects and a comparison with other clinical tests used in single and dual-task conditions. Annals of physical and rehabilitation medicine. 2009; 52 (6):453-74. Epub 2009/06/16. doi: 10.1016/j.rehab.2008.10.004 PMID: 19525161.

28. Guedes RC, Dias RC, Pereira LS, Silva SL, Lustosa LP, Dias JM. Influence of dual task and frailty on gait parameters of older community-dwelling individuals. Brazilian journal of physical therapy. 2014; 18 (5):445-52. Epub 2014/11/06. PMID: 25372007; PubMed Central PMCID: PMCPMC4228630. 